\title{
Aberrantly elevated redox sensing factor Nrf2 promotes cancer stem cell survival via enhanced transcriptional regulation of ABCG2 and Bcl-2/Bmi-1 genes
}

\author{
YAN JIA, JUN CHEN, HE ZHU, ZAN-HUI JIA and MAN-HUA CUI
}

Department of Obstetrics and Gynecology, The Second Hospital of Jilin Universty, Changchun, Jilin 130041, P.R. China

Received February 9, 2015; Accepted May 5, 2015

DOI: 10.3892/or.2015.4214

\begin{abstract}
The presence of cancer stem cells in cervical cancer stem cells (CSCs) is important in the prevention of therapy failure and tumor recurrence. Upregulation of transcriptional regulation of redox sensing factor Nrf2 leads to the overexpression of drug efflux proteins such as ABCG2 in cancer stem cells and thus results in cancer treatment failure and cancer relapse. In the present study, we purified approximately $3.1 \%$ of cervical CSCs, which exhibited aberrant upregulation of Nrf2 in conjunction with an elevated transcriptional regulation of $\mathrm{ABCG} 2, \mathrm{Bcl}-2$ and $\mathrm{Bmi}-1$. Consequently, CSCs possess prolonged cell survival, infinite cell proliferation and highly resistant apoptosis. Following silencing of the function of Nrf2 the cervical CSCs became more sensitive to DNA targeting drugs and apoptosis. Our results suggested that Nrf2-mediated drug and apoptosis resistance are important in cancer therapies and tumor recurrence. Therefore, designing anticancer drugs targeting Nrf2 may be crucial to prevent CSC-mediated tumorigenesis.
\end{abstract}

\section{Introduction}

Cervical cancer is one of the most common gynecological malignancies, resulting in a significant mortality rate in women worldwide $(1,2)$. In most cases, cervical cancer is treated by hysterectomy or primary radiotherapy after diagnosis at early stages $(1,2)$. However, patients at an advanced stage of cancer progression (regional and distant metastases) are generally treated with the combination of radio and chemotherapy. Despite the significant improvements in cancer treatment strategies, the overall survival of cervical cancer patients has yet to be determined. After treatment, $>75 \%$ of patients have recurrence within 1-2 years, resulting in tumor invasion and metastasis (3).

Correspondence to: Dr Man-Hua Cui, Department of Obstetrics and Gynecology, The Second Hospital of Jilin University, 218 Ziqiang Street, Changchun, Jilin 130041, P.R. China

E-mail: cuimanhua28@gmail.com

Key words: Nrf2, anti-apoptotic genes, drug resistance, cancer stem cells, cell proliferation
Studies conducted on different solid tumors reported that the persistence of cancer stem cells (CSCs) are the major factor responsible for therapy failure and tumor recurrence (4-7). A proposed cancer stem cell theory suggested that CSCs are a small subpopulation within the heterogenous tumor $(7,8)$ and are highly potent with regard to differentiation, infinite proliferation rate, self-renewal, tumorigenesis and invasion (7-9). Therefore, it is important to isolate and characterize CSCs to gain a better understanding of CSC-meditated tumorigenesis and metastasis. CSCs have been isolated based on their property of Hoechst 33342 dye exclusion by using fluorescence-activated cell sorting (FACS) analysis. During the FACS analysis, these CSCs were reduced on the left side of the dot plot FACS analysis quadrant and designated as side population (SP) cells (10). These SP cells share all the significant features of CSCs and elevated ATP-binding cassette (ABC) transporter protein, while ABCG2 in SP cells has been shown to be involved in Hoechst 33342 efflux or multidrug resistance (11-13). In the present study, we aimed to characterize the cervical CSCs to gain a better understanding of Nrf2-mediated drug and apoptosis resistance of CSCs.

\section{Materials and methods}

Cancer samples and cell culture. Cervical cancer samples were collected from patients at the time of surgery in the Department of Obstetrics and Gynecology in accordance with the ethical principles approved by The Second Hospital of Jilin University, China. A total of 15 samples were collected by punch biopsies. The total number of patients was 30, with an age range of 39-43. Three poorly differentiated carcinomas (PDSCC), 15 high-grade SILs and 12 well-differentiated squamous cell carcinomas (WDSCC) were identified. The corresponding control non-malignant cervical epithelial tissues were obtained from healthy groups at the time of hysterectomy. The cancer tissues were then washed and further processed as previously described (14).

FACS analysis. By using a hemocytometer, $\sim 10^{6}$ cells were taken and divided into two groups. In group I, the cells were labeled with Hoechst 33342 dye alone $(n=11)$ and in group II, the cells were treated with the reserpine drug and Hoechst 33342 dye $(n=11)$. Subsequently, the cells were counterstained with propidium iodide (PI) $2 \mu \mathrm{g} / \mathrm{ml}$ and analyzed by flow cytometer. 
Assays. Cell resistance, Matrigel invasion, sphere formation and TUNEL assays were performed exactly as previously described $(15,16)$.

Tumor cell implantation. The FACS sorted SP and non-SP cells $\left(4 \times 10^{3}\right.$ cells) were administered into NOD/SCID mice by subcutaneously injection. The density of the injected cells and mice growth was monitored as previously described (17). After 3-4 weeks, the derived tumors from the sacrificed mice were removed.

Reverse transcription (RT)-quantitative polymerase chain reaction $(q P C R)$. Total RNA was extracted and complementary DNA was prepared using Reverse Transcriptase kit (Fermentas). RT-qPCR analysis was performed on an iCycler IQ real-time detection system, using an IQ Supermix with SYBR-Green (both from Bio-Rad, Hercules, CA, USA). The sequences of human specific primers used were as follows $(15,16)$ : ABCG2 forward, TCA ATC AAA GTG CTT CTT TTT TATG and reverse, TTG TGG AAG AAT CAC GTG GC); Nrf2 forward, ACA CGG TCC ACA GCT CAT C and reverse, TGC CTC CAA AGT ATG TCA ATC A); GAPDH forward, ATG TCG TGG AGT CTA CTG GC and reverse, TGA CCT TGC CCA CAG CCT TG); Oct-4 forward, TCG AGA ACC GAG TGA GAG GC and reverse, CAC ACT CGG ACC ACA TCC TTC); Bmi-1 forward, CTCCCAACTGGTTCGACCTT and reverse, CGGTTTCCATATTTCTCAGT); CD133 forward, TCT TGA CCG ACT GAG AC and reverse, ACT TGA TGG ATG CAC CAA GCA C); EpCAM forward, CTG CCA AAT GTT TGG TGA TG and reverse, ACG CGT TGT GAT CTC CTT CT); BCL-2 forward, ACA CTG TTA AGC ATG TGC CG and reverse, CCA GCT CAT CTC ACC TCA CA); and Bax forward, GGA TGC GTC CAC CAA GAA and reverse, ACT CCC GCC ACA AAG ATG) (17-20). The PCR parameters used to set the PCR reactions were: Initial denaturation at $-95^{\circ} \mathrm{C}$ for $15 \mathrm{sec}$; annealing at $-58^{\circ} \mathrm{C}$ for $45 \mathrm{sec}$; and extension at $-60^{\circ} \mathrm{C}$ for $30-45 \mathrm{sec}$, for 35 cycles. The amplified products were visualized by ethidium bromide-stained $1.2 \%$ agarose gels. Image $\mathrm{J}$ was used to measure the band intensity. The data presented in the graph are the average values of three independent experiments.

RNA interference. Small interfering RNA (siRNA) specific to the Nrf2 gene was purchased from Dharmacon (Lafayette, CO, USA). siRNA transfection (final concentration of $200 \mathrm{~nm}$ ) was performed as per the Dharmacon protocol by using Oligofectamine 2000 (Invitrogen-Life Technologies, Carlsbad, CA, USA). The transfected siRNA cells were analyzed after $48 \mathrm{~h}$.

Immunofluorescent staining. The SP cells and main population cells were seeded in cover slips on 12 -well plates $\left(1 \times 10^{5}\right.$ cells/ well). The primary antibodies used were mouse anti-CD133 (1:100), anti-Oct-4 (1:200), anti-EpCAM (1:200), anti-ABCG2 (1:1,000), anti-bcl2 (1:100) and anti-Bax (1:200). After 24-h incubation in $1 \%$ BSA-TBS the antibodies were incubated overnight at $40^{\circ} \mathrm{C}$. After washing with $1 \mathrm{X}$ PBS, the cells were incubated with secondary antibody conjugated with FITC (dilution: 1:1,000 in 1\% BSA-TBS), at room temperature for 1-2 h. For immunohistochemistry, the tissues were processed with CD133 as previously described (21) and stained with mouse anti-CD133 (1:100) and rabbit anti-Nrf2. Tissues and cells were stained with Hoechst 33342 (dilution: 1:100; Bio-Rad) dye to visualize the nucleus.

The immunostaining of squamous spheres were performed as previously described (12). Spheres were fixed onto glass slides in ice-cold $4 \%$ paraformaldehyde $\left(40^{\circ} \mathrm{C}\right.$ for $\left.10 \mathrm{~min}\right)$, blocked with normal serum for $30 \mathrm{~min}$, and incubated with mouse monoclonal anti-Oct-4 and CD44 (1:200; Chemicon, Japan) overnight. After being washed with PBS, the slides were incubated with FITC-conjugated chicken anti-rat IgG overnight in the dark. Nuclei were counterstained with 4,6-diamidino-2-phenylindole (DAPI). The cells and tissues were subsequently viewed under a confocal laser scanning microscope (Leica TCS, Mannheim, Germany). Image analysis and figures were prepared using Adobe Photoshop CS6.

Western blot analysis. Proteins were extracted from the SP and non-SP cells, and protein concentration was determined using the Bradford assay (15). Primary antibodies (rabbit anti-human ABCG2, Nrf2 and GAPDH) were incubated overnight, followed by a secondary antibody (goat anti-rabbit IgG with alkaline phosphatase markers) and a chemiluminescence reagent. Blots were detected using gel documentation and scanned using a densitometer (GS-710; Bio-Rad).

Statistical analysis. A one-way analysis of variance (ANOVA) and Student's t-test were performed to determine the significant difference between the treatment and control groups. A probability level of $\mathrm{P}<0.05$ or 0.01 was considered statistically significant.

\section{Results}

Flow cytometry-based purification of cancer stem-like SP cells. In the FACS analysis, cells passing through the laser beam were considered forward and side scatter cells correlating with cell size and population density, respectively. Using PI staining, dead cells were excluded and the main population was gated as a P1 region. The cancer cells that efficiently effluxed the DNA binding dye Hoechst 33342 and were reduced in the left lower quadrant of the FACS profile were termed as 'side population (SP)' cells. We found $\sim 3.1 \%$ of SP cells (P2-gated population) from cervical cancer samples, which actively pumped out the Hoechst 33342 dye (Fig. 1A). The process of drug expulsion by SP cells occurred due to the overexpression of the ABC transporter protein, ABCG2. As a confirmatory test we treated the cervical cancer samples with the ABC inhibitor reserpine and analyzed using FACS. Following treatment with reserpine, the SP cell population was dramatically reduced to $0.9 \%$ (Fig. 1B). Thus, the results suggested that the presence of ABC proteins in SP cells is significant for the expulsion of DNA binding dyes.

Cervical cancer SP cells possess properties of embryonic stem cells. The FACS sorted SP and non-SP cells were further analyzed for the association of stem cell features. First, we evaluated the expression of stem cell surface genes, such as Oct-4, CD133 and EpCAM, between the SP and non-SP cells using RT-PCR analysis. As shown in the graph (Fig. 2A), the transcriptional regulation of the abovementioned genes are 

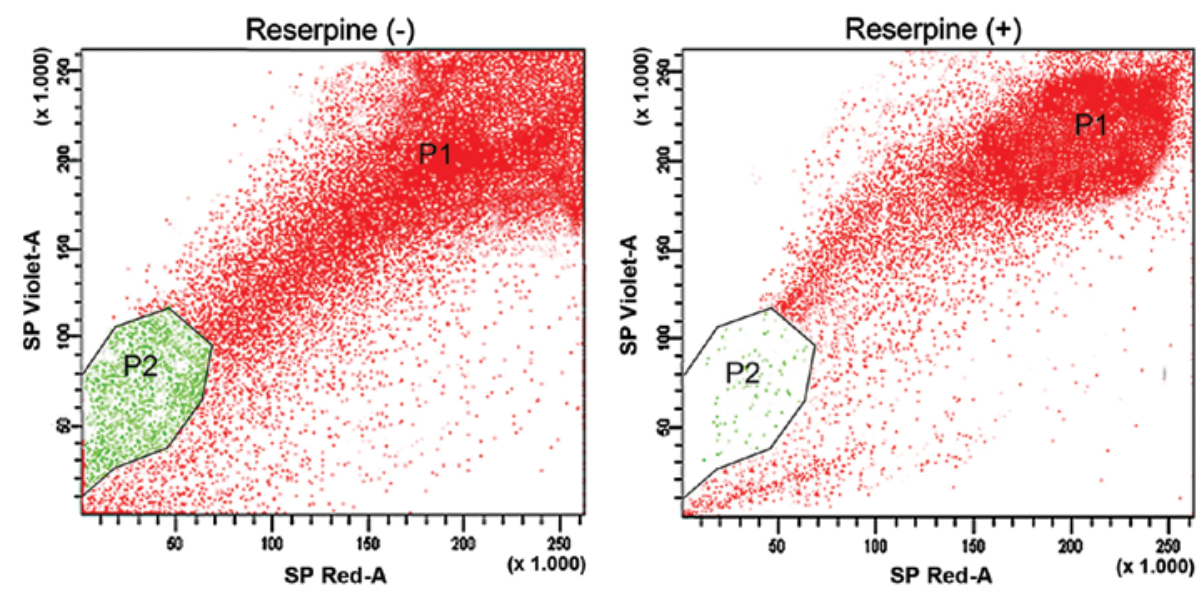

Figure 1. Purification of CSCs by FACS analysis. (A) Presence of SP cells of $\sim 3.1 \%$ from cervical cancer samples (P2 gated region). P1 indicates the main live cell population. (B) The percentage of the SP cells was significantly reduced to $0.7 \%$ following treatment with reserpine, an inhibitor of the ABC transporters CSCs, cervical cancer stem cells; SP, side population; ABC, ATP-binding cassette; FACS, fluorescence-activated cell sorting.

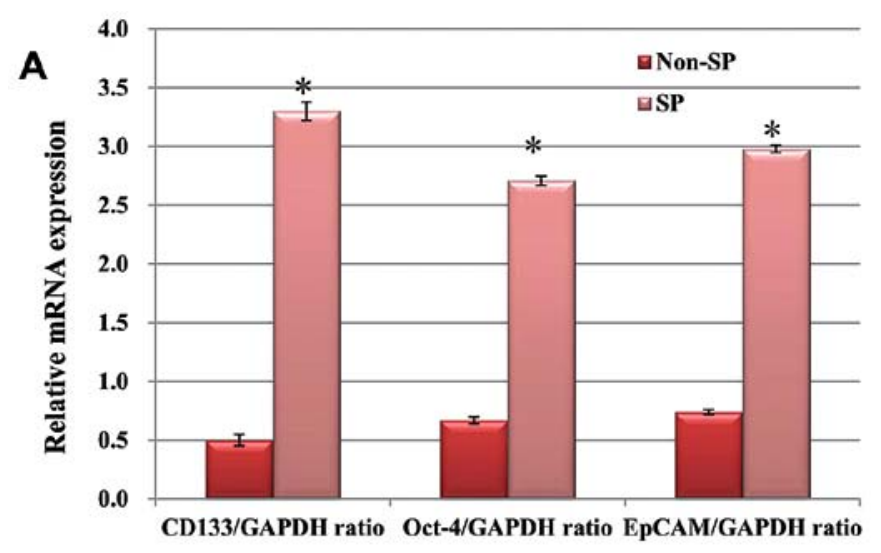

B

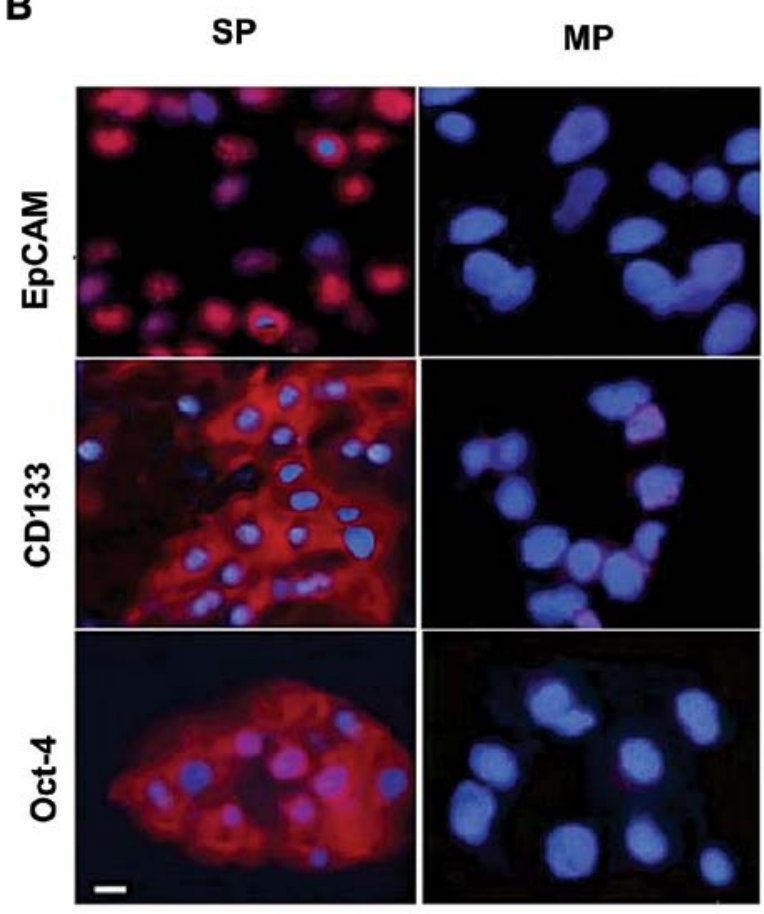

Figure 2. Enhanced stemness gene expression profile in FACS sorted SP cells. (A) Quantification graph generated from RT-PCR analysis showing upregulated mRNA transcriptional regulation stemness genes, such as CD133, EpCAM and Oct-4 in SP cells. (B) Immunocytochemistry of FACS sorted SP cells showed more positivity towards the stem cell surface proteins, whereas the non-SP cells showed either null or very weak positivity. The bar represents the standard deviation. ${ }^{*} \mathrm{P}<0.01$. Scale bar, $10 \mu \mathrm{m}$. SP, side population, FACS, fluorescence-activated cell sorting. 
A

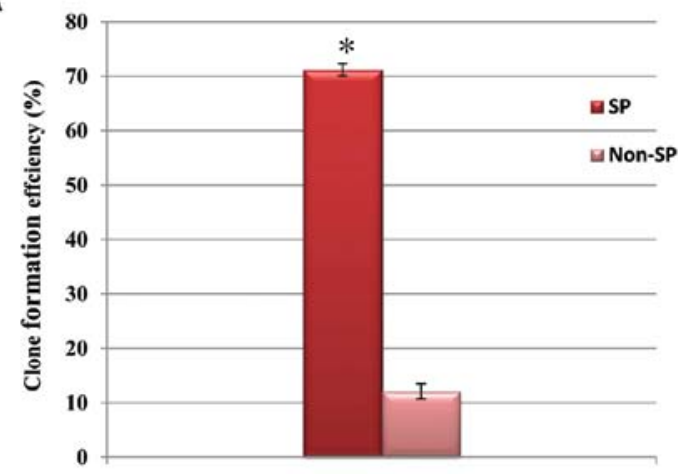

B

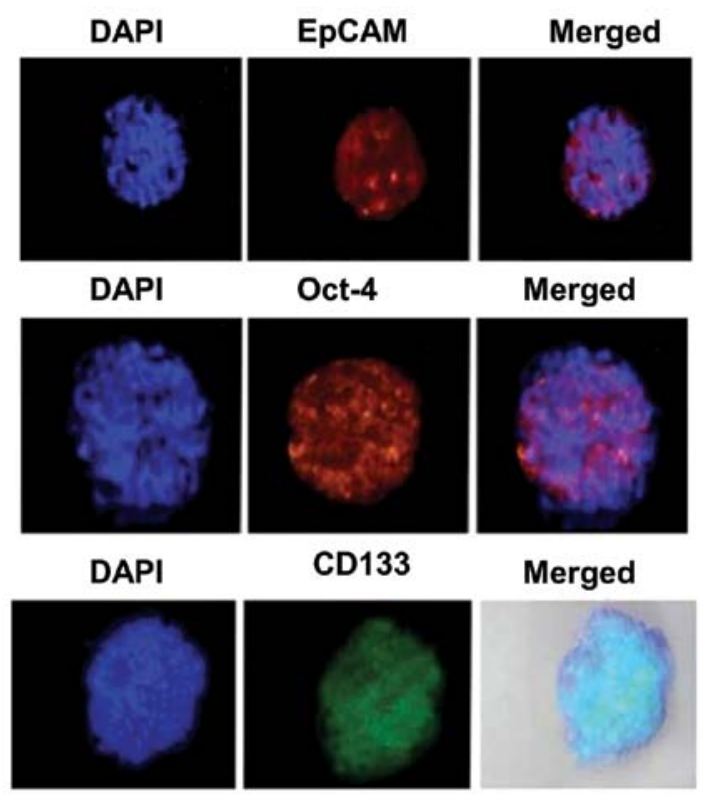

Figure 3. Cervical cancer SP cells are highly potent in generating tumor spheres. (A) Clone formation efficiency graph showing that the SP cells are rapidly generating more tumor spheres than non-SP cells. (B) Immunofluorescence staining of SP cell-induced tumor spheres exhibit positivity towards CD133, EpCAM and Oct-4. The error bar shows the standard deviation. ${ }^{*} \mathrm{P}<0.01$. SP, side population.

significantly upregulated in the SP cells than non-SP cells. In addition, our immunofluorescence assay revealed that the SP cells exhibited increased positivity towards these proteins, whereas the non-SP cells exhibited null or less positivity towards these proteins (Fig. 2B). Thus, the overexpression of these stem cell proteins may be involved in the self-renewal process. Therefore, we performed a sphere formation assay to determine the ability of self-renewal of SP cells. As expected, the SP cells produced significantly more tumor spheres and generally the tumor size was much larger than that of the SP cells (Fig. 3A). Of note, the spheres generated by the SP cells were highly positive towards stem cell surface proteins such as CD133, EpCAM and Oct-4 (Fig. 3B). Therefore, an increased expression of stemness genes in SP is crucial for the maintenance of self-renewal of the cancer stem-like SP cells.

Elevated Nrf2 attenuates apoptosis and multidrug intake. We performed a drug resistance assay to compare the cell survival rate between the SP and non-SP cells. As shown in Fig. 4A, the SP cells were more resistant to 5-fluorouracil (5-FU), cisplatin, paclitaxel and oxaliplatin. The survival rates of the SP cells were significantly higher $(>75 \%)$ following treatment with the multidrugs than the non-SP cells $(<20 \%)$. It has been shown that the aberrant upregulation of Nrf2 was involved in the regulation of $\mathrm{ABC}$ genes, thus ultimately resulting in chemotherapy resistance of the SP cells (22). Therefore, we evaluated the transcriptional expression of the Nrf2 gene in the SP and non-SP cells. The relative mRNA expression of the Nrf2 gene was significantly enhanced in the SP cells in conjunction with the elevated mRNA expression of the $\mathrm{ABC}$ transporter ABCG2 and anti-apoptotic genes such as Bcl-2 and Bmi-1 than in the non-SP cells (Fig. 4B). However, Bax gene expression was significantly reduced in the SP cells as compared to the non-SP cells. In addition, our immunofluorescence results revealed that the cervical cancer SP cells exhibited enhanced positivity towards ABCG2 and Bcl-2, but decreased positivity towards Bax proteins as compared to the non-SP cells (Fig. 5A). As a consequence of the aberrant regulation of the ABCG2 and anti-apoptotic gene expression profiles, the number of SP cells that underwent apoptosis was markedly decreased compared to that of the non-SP 


\section{A}

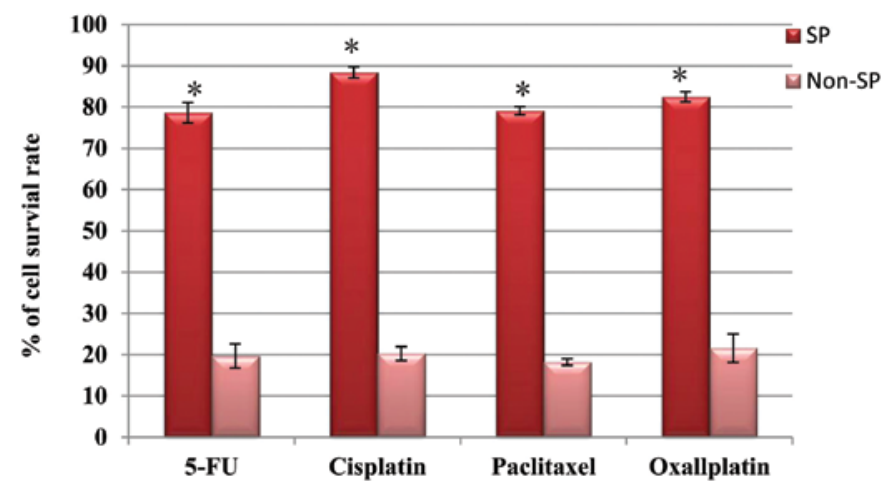

B

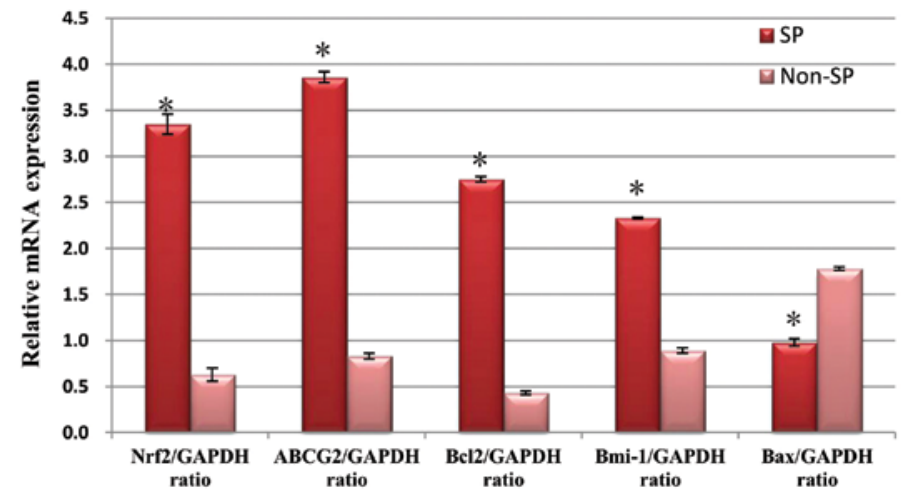

Figure 4. Elevated Nrf2 mediates enhanced cell survival of SP cells. (A) SP cells are highly resistant to 5-FU, cisplatin, paclitaxel and oxaliplatin. The SP cells have a higher survival rate $(>75 \%)$ following treatment with these drugs compared to the non-SP cells $(<20 \%)$. (B) Quantification graph generated from RT-PCR analysis showing that the relative mRNA expression profile of Nrf2 in SP cells was highly elevated in conjunction with ABCG2, Bcl-2 and Bmi-1 than in non-SP cells. Bar, standard deviation. "P<0.01. 5-FU, 5-fluorouracil; SP, side population.
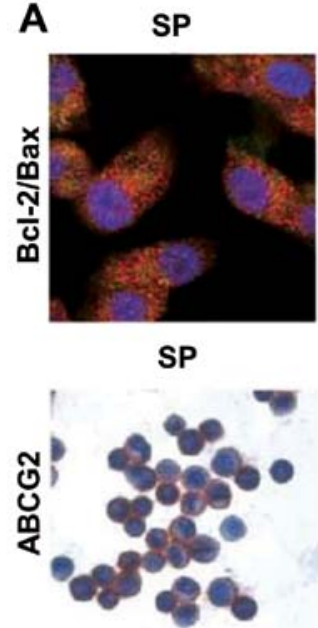

Non-SP

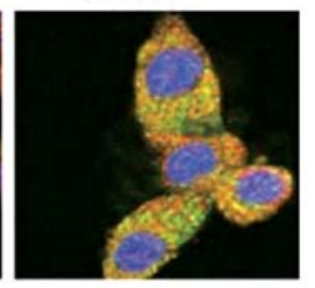

Non-SP

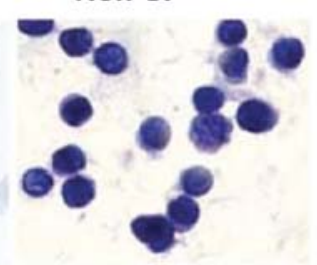

B

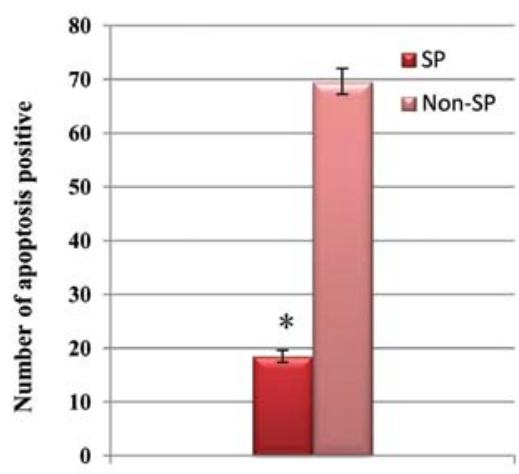

Figure 5. Elevated Nrf2 reduces programmed cell death rate in SP cells. (A) Immunofluorescence staining of SP cells showing an enhanced expression of anti-apoptotic factor Bcl-2, Bax and ABCG2 than in the non-SP cells. (B) The number of SP cells positive for apoptosis are significantly less than in the non-SP cells. Bar, standard deviation. ${ }^{*} \mathrm{P}<0.01$. SP, side population.

cells (Fig. 5B). These results suggested that the elevated expression of $\mathrm{Nrf} 2$ is crucial in the long-term survival of cancer stem cells by upregulating and downregulating the drug efflux and apoptosis mechanism, respectively.
siRNA interference of Nrf 2 enhances drug and apoptosis sensitivity of SP cells. We examined whether the rapid inactivation of Nrf2 downregulated the ABC transporter and anti-apoptotic genes. Thus, we used a silencing RNA approach to inactivate 


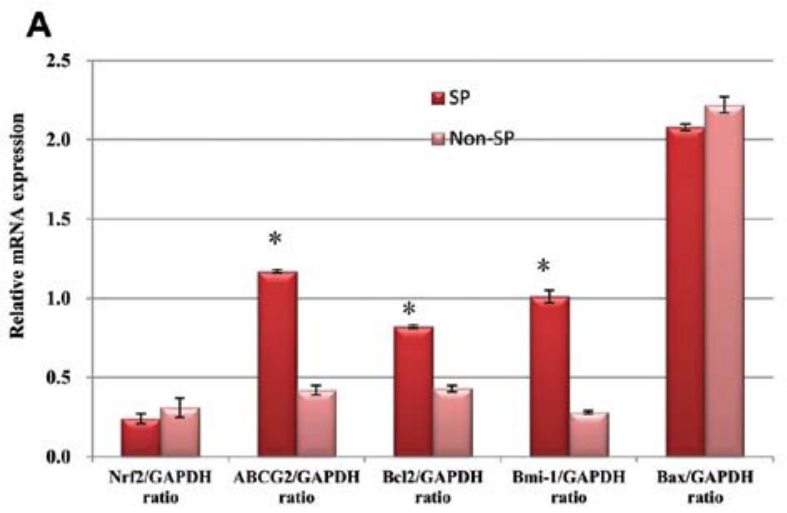

B
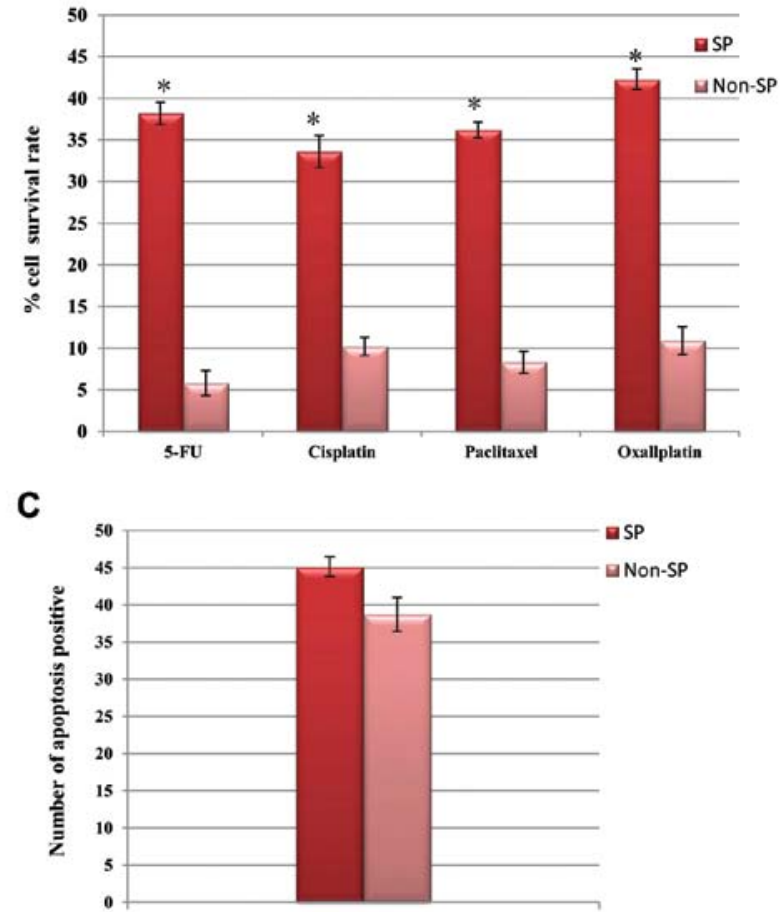

Figure 6. siRNA interference of Nrf2 enhances the drug and apoptosis sensitivity of SP cells. (A) After rapid inactivation of Nrf2, the transcriptional regulation of ABCG2, Bcl-2, Bmi-1 and Bax are significantly downregulated and upregulated, respectively. (B) Comparison of cell survival rate of SP and non-SP cells after Nrf2 knockdown. (C) Evaluation of the apoptotic rate in SP and non-SP cells in Nrf2-silenced SP and non-SP cells. Bar, standard deviation. " $\mathrm{P}<0.05$; ${ }^{* *} \mathrm{P}<0.01$. SP, side population.

the Nrf2 gene rapidly and temporarily. After silencing the Nrf2 gene in the SP cells, the transcriptional regulation of the ABCG2, Bcl-2 and Bmi-1 genes was significantly downregulated (Fig. 6A). Consequently, the SP cells were more sensitive towards multidrug treatment, as the survival rate of SP cells was significantly reduced (Fig. 6B). Similarly, the number of SP cells that underwent apoptosis was significantly elevated after Nrf2 inactivation (Fig. 6C). These results suggested that an elevated Nrf2 is a major causative factor for chemotherapy failure and poor survival rate of cancer patients.

Nrf2-upregulated SP cells are highly tumorigenic and invasive. To determine the tumorigenicity of FACS-sorted Nrf2-overexpressed SP cells, we implanted the lowest density $\left(4 \times 10^{3}\right.$ cells) of the SP and non-SP cells separately into the NOD/SCID mice. The SP cells were highly potent for initiating rapid tumor growth in the NOD/SCID mice and the tumor size was larger (Fig. 7A), whereas the non-SP cells failed to generate tumors at this cell concentration. The Matrigel invasive assay revealed that the SP cells were highly invasive (Fig. 7B). Furthermore, the tumor tissues derived from SP-induced cells showed an enhanced Nrf2 and Bcl-2 staining compared to the non-SP tissues (Fig. 8). These results clearly suggested that the presence of SP cells are the major factor involved in tumor recurrence, invasion and metastasis after chemotherapy failure.

\section{Discussion}

The heterogenous population of cancer cells contains a small proportion of tumor-initiating cells termed cancer stem cells (CSCs), responsible for multidrug therapy failure and tumor relapse as they are capable of self-renewal (23-25). CSCs have been isolated and characterized in different solid tumors such as pancreatic carcinoma, ovarian and prostate cancer (4-7). Additionally, CSCs are highly aggressive and resistant 


\section{A}
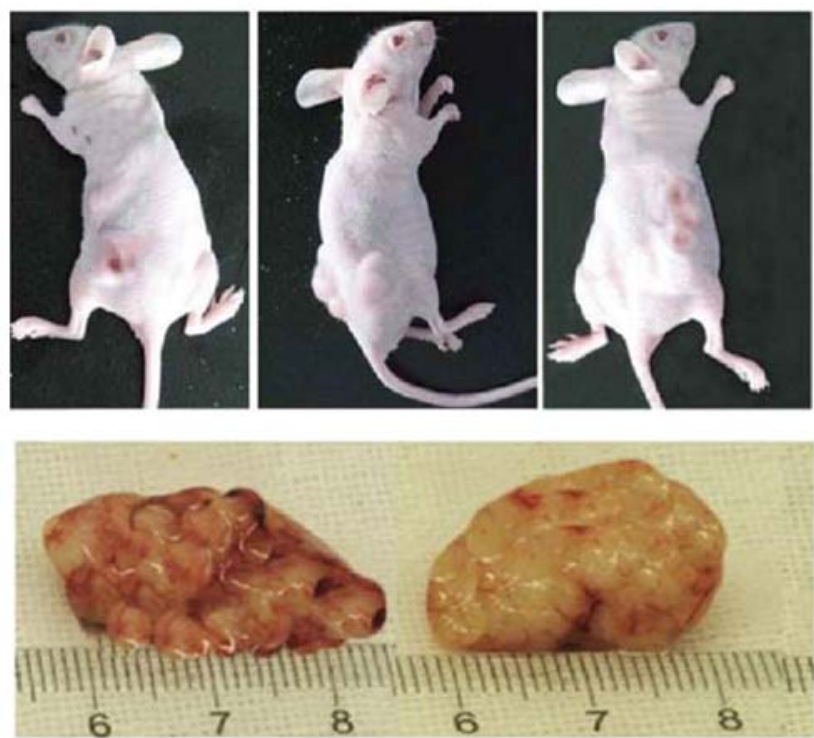

B

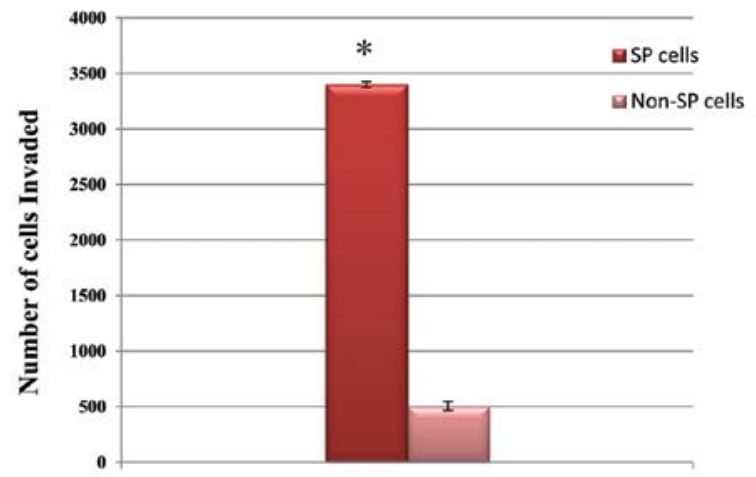

Figure 7. Nrf2 overexpression SP cells are highly tumorigenic and invasive. (A) The tumor sections derived from SP and non-SP cells after 23 days of subcutaneous injection into NOD/SCID mice. (B) Matrigel invasion assay showing the number of SP cells invading the matrigel are significantly higher than the non-SP cells. Bar, standard deviation. ${ }^{*} \mathrm{P}<0.01$. SP, side population.
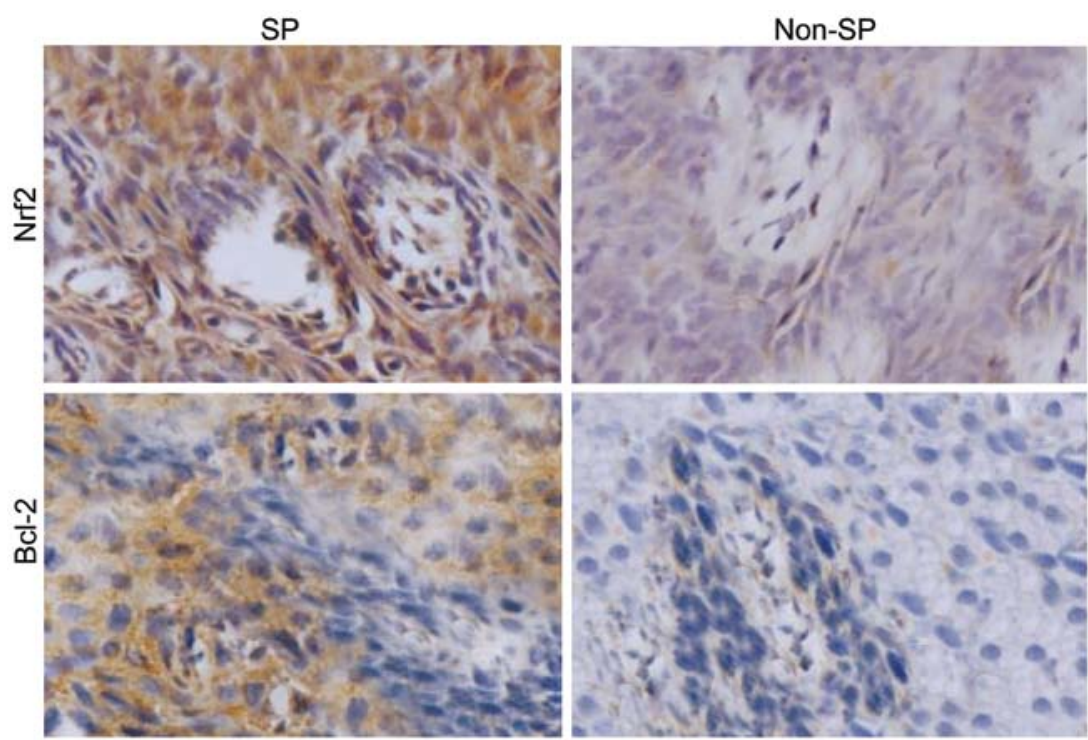

Figure 8. Immunohistochemical staining of SP and non-SP derived tumor tissues. SP cell-derived tumor tissues show enhanced positivity towards Nrf2 and Bcl-2. SP, side population. 
to multidrug treatment and apoptosis (4-7). The increased multidrug resistance properties of CSCs are mainly due to the higher expression levels of drug efflux pumps such as $\mathrm{ABC}$ transporters ABCG2 and Bcrp1 which are encoded by multidrug-resistant (MDR) gene 1 (12). However, investigations into CSCs to identify novel signaling and aberrantly upregulated proteins in CSCs may be useful to developing novel and effective anticancer drugs.

In the present study, by using the Hoechst 33342 dye exclusion assay we have isolated and characterized the cancer stem-like SP cells from cervical cancer samples. We have found a significant amount (3.1\%) of cervical cancer SP cells. The SP cell population possesses overexpression of $\mathrm{ABC}$ transporter proteins such as ABCG2. Similarly, it has been previously showed in cervical cancer that HeLa stem cells possess the highly expressed MDR protein Bcrp1 (3). Furthermore, we have demonstrated that FACs-sorted cervical cancer SP cells are capable of self-renewal as they express higher levels of stem cell surface proteins such as CD133, CD44, EpCAM and Oct-4, which are involved in the maintenance of self-renewal. These proteins are also responsible for the higher cell proliferation rate and rapid generation of tumor spheres because the FACs-purified SP cells are able to initiate tumor spheres. These tumor spheres are highly positive for CD133, CD44 and Oct-4. With regard to these findings, it has been previously shown that $\mathrm{Bcrp}^{+}$cells underwent rapid proliferation and they are capable of self-renewal $(3,26)$. Another interesting phenotype of the SP cells we identified is that they are highly resistant to multidrugs and apoptosis. Furthermore, these SP cells can initiate tumor growth in NOD/SCID mice even at the lowest cell concentration, confirming that SP cells are capable of causing tumor recurrence and invasion following therapy failure. Similarly, the $\mathrm{Bcrp}^{+} / \mathrm{CD} 44^{+}$cervical cancer SP cells are highly tumorigenic and induce rapid tumor growth in NOD/SCID mice (9). Overexpression of the stem cell surface protein CD133 in different types of cancer has been shown to be involved chemoresistance, self-renewal and tumorigenesis in vitro and in vivo (27). However, the signaling mechanism and downstream pathways involved in drug resistance, apoptosis resistance and tumor recurrence remain to be determined.

The major role of Nrf2 is providing protection to the cells from oxidative stress and other environmental cues, such as xenobiotics. Generally, the downstream signaling of Nrf2 leads to activation of several antioxidant enzymes and an elevated expression of various drug efflux transporter proteins on the cell surface (28-30). On the other hand, negative regulation of Nrf2 leads to ubiquitin-mediated proteosomal degradation by KEAP1 $(31,32)$. Loss of Nrf2-KEAP1 interaction or point mutations in KEAP1 has been found to lead to the upregulation of the Nrf2 gene, which is often present in primary tumors $(33,34)$. Studies in lung cancer stem cells reported that upregulated $\mathrm{Nrf} 2$ leads to the overexpression of $\mathrm{ABC}$ transporter proteins, contributing to chemotherapy failure $(2,12,17)$. Furthermore, depletion of Nrf2 leads to the suppression of $\mathrm{ABC}$ transporter gene expression and its function (22). Similarly, we have demonstrated that cervical cancer SP cells possess an aberrantly elevated level of the Nrf2 transcript and consequently the transcriptional regulation of $\mathrm{ABCG} 2$ and $\mathrm{Bcl} 2 / \mathrm{Bmi}-1$ are highly upregulated. Furthermore, we have shown that silencing of the Nrf2 gene expression attenuated the expression of ABCG2, Bcl-2 and Bmi-1, rendering the SP cells more sensitive to drug treatment and efficiently subjected to apoptosis. There is a high risk of alteration in programmed cell death during carcinogenesis associated with mutation in the p53 gene and modulation of apoptotic regulatory Bcl-2, Bmi-1 and Bax proteins in cervical CSCs (18). Inactivation of the p53 gene induces the production of other pro-apoptotic proteins such as Bax and represses the anti-apoptotic factors such as Bcl-2 and Bmi-1 (18). Therefore, we hypothesized that an elevated level of Nrf2 may be involved in the inactivation of the p53 gene and its function.

Taken together, our results suggest that elevated Nrf2 signaling in cervical cancer SP cells are a major root cause for DNA targeting drug and cell death resistance. Future studies concerning elucidation of the precise molecular mechanism behind the regulatory pathways and additional factors involved in Nrf2-mediated ABCG2/Bcl-2 overexpression may provide insight into the development of novel anticancer drugs that effectively suppress Nrf2 signaling and the function of drug efflux pumps.

\section{Acknowledgements}

The present study is supported by the Youth Fund of Science and Technology Department, Jilin (no. 201201034). We would like to thank Dr Wanshan Li (Department of Oral and Maxillofacial Surgery, Children's Hospital, Chongqing Medical University); Dr Yang Liu (Department of Thoracic Surgery, Chinese PLA General Hospital); Dr Nan Jiang (Department of Hepatic Surgery, The Third Affiliated Hospital of Sun Yat-Sen University); Dr Yang Liu, (Department of Thoracic Surgery, Chinese PLA General Hospital); and Dr Xue-Hui Wang (Department of Gynecology, The Affiliated Zhongshan Hospital of Dalian University) for their helpful guidance, collaboration and sharing of all the important protocols and primers.

\section{References}

1. Bansal N, Herzog TJ, Shaw RE, Burke WM, Deutsch I and Wright JD: Primary therapy for early-stage cervical cancer: Radical hysterectomy vs radiation. Am J Obstet Gynecol 201: 485.e1-485.e9, 2009.

2. Kosmas C, Mylonakis N, Tsakonas G, Vorgias G, Karvounis N, Tsavaris N, Daladimos T, Kalinoglou N, Malamos N, Akrivos T, et al: Evaluation of the paclitaxel-ifosfamide-cisplatin (TIP) combination in relapsed and/or metastatic cervical cancer. Br J Cancer 101: 1059-1065, 2009.

3. Zhang SL, Wang YS, Zhou T, Yu XW, Wei ZT and Li YL: Isolation and characterization of cancer stem cells from cervical cancer HeLa cells. Cytotechnology 64: 477-484, 2012.

4. Bapat SA, Mali AM, Koppikar CB and Kurrey NK: Stem and progenitor-like cells contribute to the aggressive behavior of human epithelial ovarian cancer. Cancer Res 65: 3025-3029, 2005.

5. Collins AT, Berry PA, Hyde C, Stower MJ and Maitland NJ: Prospective identification of tumorigenic prostate cancer stem cells. Cancer Res 65: 10946-10951, 2005.

6. Olempska M, Eisenach PA, Ammerpohl O, Ungefroren H, Fandrich F and Kalthoff H: Detection of tumor stem cell markers in pancreatic carcinoma cell lines. Hepatobiliary Pancreat Dis Int 6: 92-97, 2007.

7. Tu SM, Lin SH and Logothetis CJ: Stem-cell origin of metastasis and heterogeneity in solid tumours. Lancet Oncol 3: 508-513, 2002 . 
8. Szotek PP, Pieretti-Vanmarcke R, Masiakos PT, Dinulescu DM, Connolly D, Foster R, Dombkowski D, Preffer F, Maclaughlin DT and Donahoe PK: Ovarian cancer side population defines cells with stem cell-like characteristics and Mullerian inhibiting substance responsiveness. Proc Natl Acad Sci USA 103: 11154-11159, 2006.

9. Al-Hajj M, Wicha MS, Benito-Hernandez A, Morrison SJ and Clarke MF: Prospective identification of tumorigenic breast cancer cells. Proc Natl Acad Sci USA 100: 3983-3988, 2003.

10. Goodell MA, Brose K, Paradis G, Conner AS and Mulligan RC: Isolation and functional properties of murine hematopoietic stem cells that are replicating in vivo. J Exp Med 183: 1797-1806, 1996.

11. Zhou S, Schuetz JD, Bunting KD, Colapietro AM, Sampath J, Morris JJ, Lagutina I, Grosveld GC, Osawa M, Nakauchi H, et al: The ABC transporter Bcrp1/ABCG2 is expressed in a wide variety of stem cells and is a molecular determinant of the side-population phenotype. Nat Med 7: 1028-1034, 2001.

12. Hirschmann-Jax C, Foster AE, Wulf GG, Nuchtern JG, Jax TW, Gobel U, Goodell MA and Brenner MK: A distinct 'side population' of cells with high drug efflux capacity in human tumor cells. Proc Natl Acad Sci USA 101: 14228-14233, 2004.

13. Wulf GG, Wang RY, Kuehnle I, Weidner D, Marini F, Brenner MK, Andreeff M and Goodell MA: A leukemic stem cell with intrinsic drug efflux capacity in acute myeloid leukemia. Blood 98: 1166-1173, 2001.

14. Qin ZL, Zheng XX and Xu YH: Increased angiogenesis and decreased programmed cell death increases the risk of uterine cervical cancer. Drug Res: Oct 21, 2014 (Epub ahead of print).

15. Yang B, Ma YF and Liu Y: Elevated expression of Nrf-2 and ABCG2 involved in multi-drug resistance of lung cancer SP cells. Drug Res: Nov 4, 2014 (Epub ahead of print).

16. Zhang QH, Dou HT, Xu P, Zhuang SC and Liu PS: Tumor recurrence and drug resistance properties of side population cells in high grade ovary cancer. Drug Res 65: 153-157, 2015.

17. Shi Y, Fu X, Hua Y, Han Y, Lu Y and Wang J: The side population in human lung cancer cell line NCI-H460 is enriched in stem-like cancer cells. PLoS One 7: e33358, 2012.

18. Fan J, Li R, Zhang R, Liu HL, Zhang N, Zhang FQ and Dou KF: Effect of Bcl-2 and Bax on survival of side population cells from hepatocellular carcinoma cells. World J Gastroenterol 13: 6053-6059, 2007.

19. Park JR, Kim RJ, Lee YK, Kim SR, Roh KJ, Oh SH, Kong G, Kang KS and Nam JS: Dysadherin can enhance tumorigenesis by conferring properties of stem-like cells to hepatocellular carcinoma cells. J Hepatol 54: 122-131, 2011.

20. Shimamura T, Yasuda J, Ino Y, Gotoh M, Tsuchiya A, Nakajima A, Sakamoto M, Kanai Y and Hirohashi S: Dysadherin expression facilitates cell motility and metastatic potential of human pancreatic cancer cells. Cancer Res 64: 6989-6995, 2004

21. Kim RJ, Kim SR, Roh KJ, Park SB, Park JR, Kang KS, Kong G, Tang B, Yang YA, Kohn EA, et al: Ras activation contributes to the maintenance and expansion of Sca- $1^{\text {pos }}$ cells in a mouse model of breast cancer. Cancer Lett 287: 172-181, 2010
22. Singh A, Wu H, Zhang P, Happel C, Ma J and Biswal S: Expression of ABCG2 (BCRP) is regulated by Nrf2 in cancer cells that confers side population and chemoresistance phenotype. Mol Cancer Ther 9: 2365-2376, 2010.

23. Bonnet D and Dick JE: Human acute myeloid leukemia is organized as a hierarchy that originates from a primitive hematopoietic cell. Nat Med 3: 730-737, 1997.

24. Lapidot T, Sirard C, Vormoor J, Murdoch B, Hoang T, Caceres-Cortes J, Minden M, Paterson B, Caligiuri MA and Dick JE: A cell initiating human acute myeloid leukaemia after transplantation into SCID mice. Nature 367: 645-648, 1994.

25. Reya T, Morrison SJ, Clarke MF and Weissman IL: Stem cells, cancer, and cancer stem cells. Nature 414: 105-111, 2001.

26. Hass R, Giese G, Meyer G, Hartmann A, Dörk T, Köhler L, Resch K, Traub P and Goppelt-Strübe M: Differentiation and retrodifferentiation of U937 cells: Reversible induction and suppression of intermediate filament protein synthesis. Eur J Cell Biol 51: 265-271, 1990.

27. Salnikov AV, Gladkich J, Moldenhauer G, Volm M, Mattern J and Herr I: CD133 is indicative for a resistance phenotype but does not represent a prognostic marker for survival of non-small cell lung cancer patients. Int J Cancer 126: 950-958, 2010.

28. Itoh K, Chiba T, Takahashi S, Ishii T, Igarashi K, Katoh Y, Oyake T, Hayashi N, Satoh K, Hatayama I, et al: An Nrf2/small Maf heterodimer mediates the induction of phase II detoxifying enzyme genes through antioxidant response elements. Biochem Biophys Res Commun 236: 313-322, 1997.

29. Ramos-Gomez M, Kwak MK, Dolan PM, Itoh K, Yamamoto M, Talalay P and Kensler TW: Sensitivity to carcinogenesis is increased and chemoprotective efficacy of enzyme inducers is lost in nrf 2 transcription factor-deficient mice. Proc Natl Acad Sci USA 98: 3410-3415, 2001.

30. Kwak MK, Kensler TW and Casero RA Jr: Induction of phase 2 enzymes by serum oxidized polyamines through activation of Nrf2: Effect of the polyamine metabolite acrolein. Biochem Biophys Res Commun 305: 662-670, 2003.

31. Kobayashi A, Kang MI, Okawa H, Ohtsuji M, Zenke Y, Chiba T, Igarashi $\mathrm{K}$ and Yamamoto M: Oxidative stress sensor Keap1 functions as an adaptor for Cul3-based E3 ligase to regulate proteasomal degradation of Nrf2. Mol Cell Biol 24: 7130-7139, 2004.

32. Zhang DD, Lo SC, Cross JV, Templeton DJ and Hannink M: Keap1 is a redox-regulated substrate adaptor protein for a Cul3-dependent ubiquitin ligase complex. Mol Cell Biol 24: 10941-10953, 2004.

33. Singh A, Misra V, Thimmulappa RK, Lee H, Ames S, Hoque MO, Herman JG, Baylin SB, Sidransky D, Gabrielson E, et al: Dysfunctional KEAP1-NRF2 interaction in non-small-cell lung cancer. PLoS Med 3: e420, 2006.

34. Shibata T, Ohta T, Tong KI, Kokubu A, Odogawa R, Tsuta K, Asamura H, Yamamoto $M$ and Hirohashi S: Cancer related mutations in NRF2 impair its recognition by Keap1-Cul3 E3 ligase and promote malignancy. Proc Natl Acad Sci USA 105: 13568-13573, 2008. 